\title{
The Influence of Strain-Optic Coefficients on the Transduction Mechanism of Planar Glass Etalon Fabry-Pérot Ultrasound Sensors
}

\author{
Danny R. Ramasawmy*, Ben T. Cox and Bradley E. Treeby \\ Department of Medical Physics and Biomedical Engineering, University College London, London, UK \\ *danny.ramasawmy.15@ucl.ac.uk
}

\begin{abstract}
The Fabry-Pérot (FP) ultrasound sensor can detect ultrasound over a broadband frequency range (tens of $\mathrm{MHz}$ ) with small element sizes (tens of microns). The FP sensor is an optical interferometer where an incident acoustic wave modulates the optical path length via two mechanisms. The first is from a change in the optical-cavity thickness and the second is from a straininduced change in refractive index. One way to investigate the transduction mechanisms is to examine the sensor's frequencydependent directional response. Previously, the strain-optic effect was neglected when modeling the directional response, however, some experimental studies have shown it to be significant in some circumstances. In this paper, the contributions of the two mechanisms are investigated for a planar air-backed glass-etalon Fabry-Pérot sensor. The sensor was modeled as a multilayered elastic structure, and the strain and displacement fields were calculated using the partial wave method. For an optically isotropic, homogeneous and non-absorbing spacer material the strain-optic effect is only dependent on two coefficients, $p_{11}$ and $p_{12}$. The modeled directivity was similar when including or excluding the strain-optic coefficients and had good agreement with directional response measurements made on an air-backed glass-etalon sensor. The directional response is dominated by the normal strain in the sensor except at the compressional critical angle where the transverse strain is larger than normal strain. Understanding how the strain-optic mechanism affects the directional response can help improve future sensor design. For example, exploiting materials with large strain-optic coefficients could increase the sensitivity of Fabry-Pérot sensors.
\end{abstract}

\section{INTRODUCTION}

The Fabry-Pérot (FP) ultrasound sensor can detect ultrasound over a broadband frequency range (tens of $\mathrm{MHz}$ ) with small element sizes (tens of microns). It has frequently been used for photoacoustic imaging and general ultrasound field characterization and as a reference sensor for hydrophone calibration [1]-[8]. The FP ultrasound sensor detects ultrasound using interferometry, where an incident acoustic wave modulates the optical path length via two mechanisms. The first is from a change in the optical-cavity thickness and the second is from a strain-induced change in refractive index. This is often referred to as the strain-optic or photo-elastic effect. The transduction mechanism of the FP sensor can

This work was supported in part by the Engineering and Physical Sciences Research Council (EPSRC), UK, Grant Nos. EP/P008860/1, EP/L020262/1 and EP/S026371/1, in part by the EPSRC-funded UCL Centre for Doctoral Training in Medical Imaging (EP/L016478/1) and the Department of Healths NIHR-funded Biomedical Research Centre at NIHR Biomedical Research Centre at University College London Hospitals. be investigated by measuring and modeling the frequencydependent directional response of the sensor (this is described further in Section II). The directional response of the FP sensor is complicated due to the complex wave-field produced within each layer of the sensor when an acoustic wave passes though it. The response is made more complex when accounting for refractive index changes. Previous models of the directional response for planar FP sensors have neglected the strain-optic mechanism and obtained good agreement with measurements [4], [9]. However, some models of FP fiber-optic hydrophone (FOH) sensors have shown the strain-optic effect to be a necessary inclusion when modeling the frequency-response [3], [10]. In this study, the contribution of the strain-optic effect on the directional response is investigated for a planar glass-etalon sensor.

\section{Model of the FAbry-PÉrot Ultrasound SEnsor}

\section{A. Transduction Mechanism}

The FP sensor was constructed from a glass microscope cover-slip $(175 \mu \mathrm{m})$, which had thin partially-reflecting aluminium mirrors $\left(<1 \times 10^{-8} \mathrm{~m}\right)$ deposited on either side. The cover-slip was mounted at the edges to a polycarbonate frame which gave the cover-slip an air-backing. Other planar FP sensors are commonly deposited onto a glass or polycarbonate substrate. The material properties of this sensor can be seen in Table I. An illustration of the air-backed cover-slip FP sensor can be seen in Fig. 1. The FP is interrogated at the base by a collimated laser beam. Light from the laser is multiply reflected by the aluminium mirrors and the intensity of the reflected light is measured. When an acoustic wave propagates through the sensor, the intensity of the reflected laser light is modulated through two mechanisms. The first mechanism is a physical change in the optical path length as the distance between the two mirrors is modulated. This is calculated by taking the difference in the vertical component of the displacement field, $u_{z}$, at the top and bottom mirrors. The second mechanism is a change in the refractive index $\Delta n$ of the cover-slip caused by local changes in density. The glass cover-slip is assumed to be optically isotropic, homogeneous and non-absorbing. If the interrogation laser beam is parallel to 
TABLE I

TABle of Material Properties

\begin{tabular}{|c|ccc|}
\hline Material & $c_{l}\left(\mathrm{~ms}^{-1}\right)$ & $c_{s}\left(\mathrm{~ms}^{-1}\right)$ & $\rho\left(\mathrm{kgm}^{-3}\right)$ \\
\hline Water [12] & 1448 & 0 & 1000 \\
Glass [12] & 5570 & 3430 & 2500 \\
Air [12] & 330 & 0 & 1 \\
Aluminium [12] & 6250 & 3100 & 2700 \\
\hline
\end{tabular}

$c_{l}, c_{s}$ : compressional and shear sound speeds, $\rho$ : density.

the $z$-axis and polarized in the $x$-axis, the change in refractive index can be written

$$
\Delta n=-\frac{1}{2} n_{0}^{3}\left(p_{11} \frac{\partial u_{x}}{\partial x}+p_{12} \frac{\partial u_{z}}{\partial z}\right) .
$$

Here, $n_{0}$ is the refractive index of the glass cover-slip and $p_{11}$ and $p_{12}$ are strain-optic coefficients (SOCs) from the $6 \times 6$ strain-optic tensor (see [11]). If the incident laser light is parallel to $z$ and polarized in $y$, the SOC $p_{11}$ is replaced with $p_{12}$. The $p_{12}$ SOC is multiplied by the normal strain in the $z$-axis and the $p_{11}$ SOC is multiplied by the normal strain in the $x$-axis. For clarity, the normal strain in the $x$-axis will be referred to as the transverse strain. The SOCs for a few glassy materials have been given in Table II. The sensor presented here is constructed from fused-silica (7070). Assuming the interrogation spot-size of the laser beam is small enough to neglect the effect of spatial averaging, the frequency-dependent directional response $D(f, \theta)$ can be written [9]

$$
D(f, \theta) \propto\left(n_{0}\left(u_{z}\left(z_{2}\right)-u_{z_{2}}\left(z_{1}\right)\right)+\int_{z_{1}}^{z_{2}} \Delta n . d z\right) .
$$

As the aluminium mirrors are orders of magnitude thinner than the cover-slip, the mirrors can be considered infinitesimally thin and the acoustic phase changes associated with the mirrors can accounted for by increasing the thickness of the coverslip [8]. Additionally, since the aluminium mirrors are highly reflective for the optical interrogation wavelength used here, the contribution of the strain-induced refractive index changes in the coupling fluid can be considered much smaller than the interference effect within the cover-slip. Hence, just the strain-optic effect within the cover-slip needs to be considered [8]. This is not necessarily the case for multi-layered dielectric mirrors, which are thicker and have different refractive indices and SOCs for each material layer [3], [6], [10].

\section{B. Modelling the Elastic Deformation}

As mentioned in the previous section, the displacement of the mirrors and the strain within the sensor is needed to calculate the frequency-dependent directional response. The multi-layered elastic structure of the FP sensor can be modeled using matrix methods. These approximate the displacement and stress fields within each layer as a sum of partial waves corresponding to multiply reflected compressional and shear waves. A three-layer elastic model was used to model the cover-slip sensor. These layers were: water (coupling fluid), the glass cover-slip and air backing. The displacement field

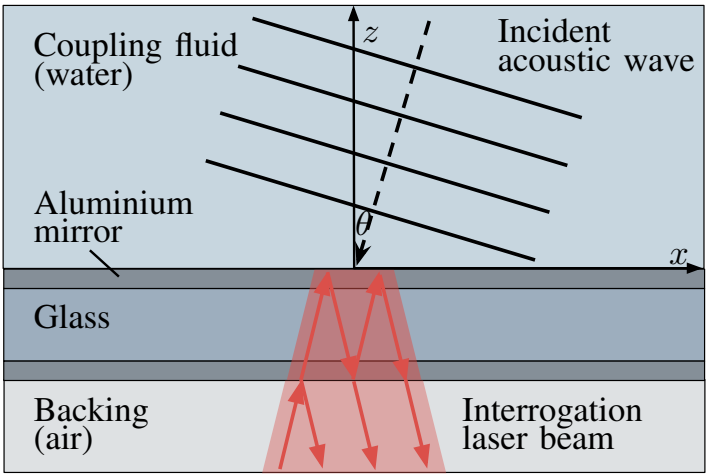

Fig. 1. Diagram of the air-backed cover-slip Fabry-Pérot ultrasound sensor. Two aluminium mirrors are separated by a glass cover-slip. Light from an interrogating laser is multiply reflected between the two mirrors and the intensity of the reflected light is measured. Note, the interrogation laser beam is parallel to the $z$-axis. An incident acoustic wave modulates the distance between the two mirrors and the strain-optic coefficients.

TABLE II

TABLE OF STRAIN-OPTIC COEFFICIENTS

\begin{tabular}{|c|ccc|}
\hline Material & $p_{11}$ & $p_{12}$ & $n_{0}$ \\
\hline $\mathrm{SiO}_{2} /$ Quartz [10], [15] & 0.121 & 0.27 & 1.456 \\
Fused silica (7940) [16] & 0.126 & 0.26 & 1.457 \\
Fused silica (7070) [16] & 0.113 & 0.23 & 1.469 \\
PMMA (Plexiglass 55) [17], [18] & 0.300 & 0.297 & 1.49 \\
\hline
\end{tabular}

within the sensor was used to calculate the normal and transverse strain and the difference in vertical displacements of the mirrors. Additionally, dispersion curves were extracted from the model to interpret the features in the directivity. For more details about the method see [4], [9], [13].

\section{Measurements of the Directional Response}

The model was compared to directional response measurements made on the air-backed cover-slip FP sensor. A short description of the method will be given here, the full experimental setup can be found in [14]. The sensor was placed at the base of a water bath suspended above the optics needed to interrogate the FP sensor. A broadband (up-to 80 $\mathrm{MHz}$ ) planar photo-acoustic source was attached to a rotating arm and stepped in $0.25^{\circ}$ increments about the interrogation point on the surface of the FP sensor. For every angle (between $\pm 40^{\circ}$ ), a time series measurement was taken and Fouriertransformed. Each angle was normalized by the frequencyresponse at normal-incidence.

\section{ANALYSIS}

\section{A. Features of the Directional Response}

The modelled directional response had good agreement with the measured data both with and without the inclusion of the strain-optic mechanism. The difference in the predictions of the models was within the uncertainty of the measurements. The majority of features in the directional response for the airbacked cover-slip can be associated with Lamb modes. These are the fundamental and higher order symmetric $(\mathrm{S} 0, \mathrm{~S} 1, \mathrm{~S} 2)$ and anti-symmetric Lamb modes $(\mathrm{A} 0, \mathrm{~A} 1, \mathrm{~A} 2)$ [9]. This can 


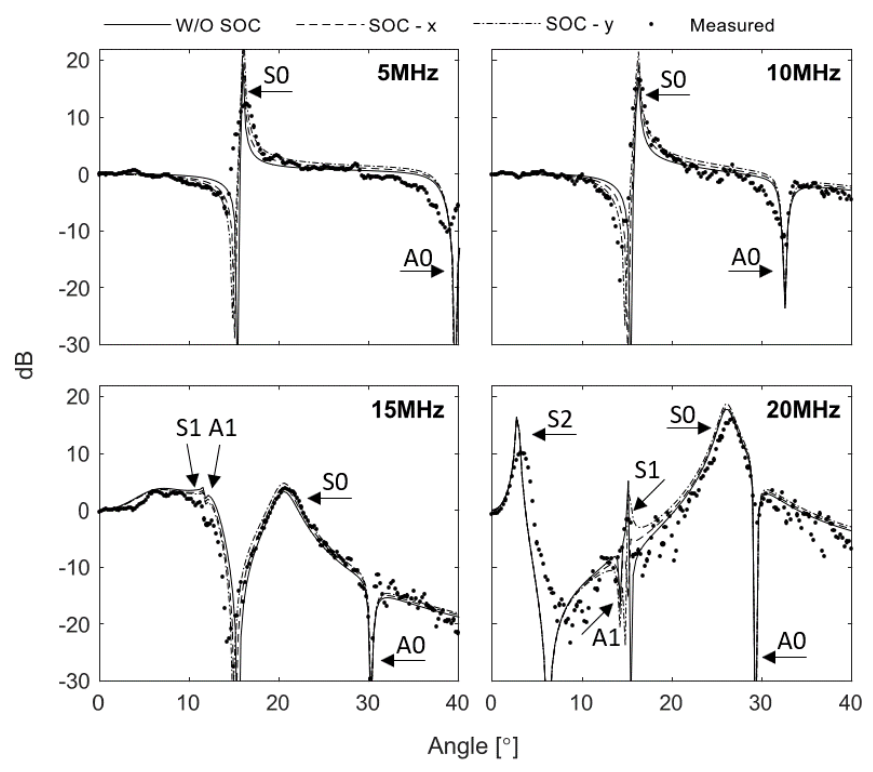

Fig. 2. Profiles of the measured and modeled directional response at four frequencies: $5 \mathrm{Mhz}, 10 \mathrm{MHz}, 15 \mathrm{MHz}$ and $20 \mathrm{MHz}$. Profiles of the measured data (points), modeled directivity excluding (solid) and including the SOCs have been plotted (polarized in $x$ - dashed, polarized in $y$ - dash-dotted). Features in the directional response correspond to the fundamental and higherorder symmetric (S) and anti-symmetric (A) Lamb modes.

be seen in Fig. 2. Profiles of the measured and modeled directional response for four frequencies have been plotted. The profiles have been normalized to the normal incidence frequency-response. As the directional response is symmetric about $0^{\circ}$ the profiles have been plotted from $0^{\circ}$ to $40^{\circ}$. Each sub-plot contains four profiles, the model without the SOCs (solid), the model including SOCs, polarized in $x$ (dashed) and $y$ (dash-dotted) and the measured response (points). The modeled directivity profiles at $10 \mathrm{MHz}$ have been plotted in Fig. 3 for clarity in comparing the model with and without SOCs. The vertical dashed line at $\theta=15.4^{\circ}$ corresponds to the compressional wave critical angle between water and glass.

At the critical angle there is only a compressional wave traveling perpendicular to the interfaces within the glass coverslip. If the SOCs are excluded, the directional response is only dependent on the vertical displacement of the mirrors. At the critical angle there is no vertical displacement and therefore a minima in the response. This can be seen in Fig. 3 (solid line). When the SOCs are included, the sensor is sensitive at the critical angle as there is a transverse strain component which effectively shifts the minima associated with the critical angle towards $0^{\circ}$. This can be seen in Fig. 3 (solid black and dashed lines). The minima is shifted to the left as the transverse strain component increases, peaking at the fundamental symmetric mode, $S 0$ which is excited just after the critical angle. The Lamb modes produce large normal and transverse strains within the cover-slip when they are excited. This can be seen in Fig. 4 which shows the normal (a) and transverse (b) strain components integrated over the coverslip thickness. These terms are important in the directivity

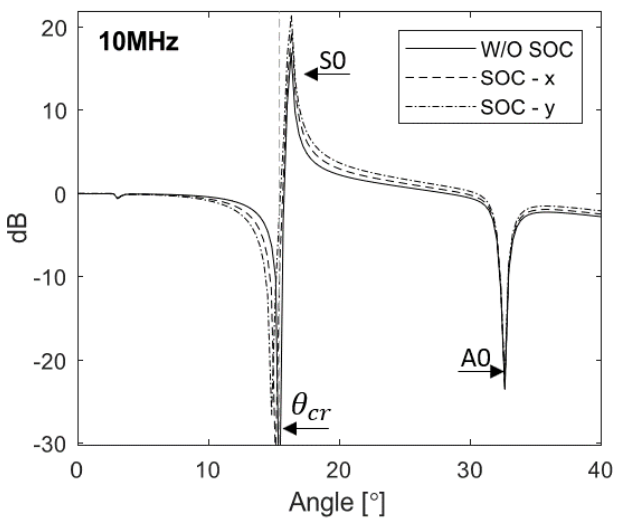

Fig. 3. Profiles of the modeled directional response at $10 \mathrm{MHz}$. Modeled directivity excluding (solid) and including the SOCs have been plotted (polarized in $x$-dashed, polarized in $y$ - dash-dotted).

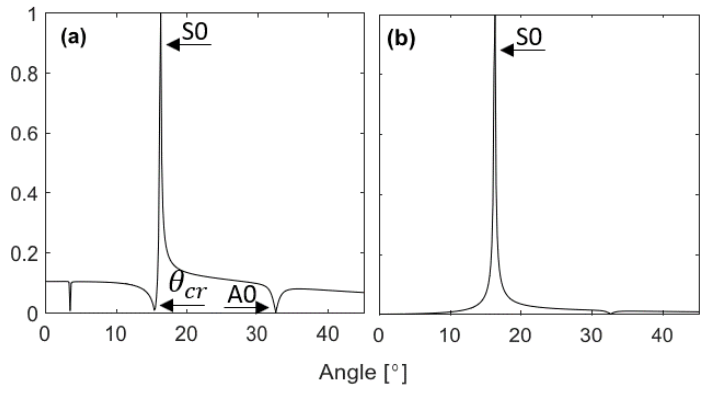

Fig. 4. (a) Integral of the normal strain component over the spacer thickness, $\int\left(\partial u_{z} / \partial z\right) \cdot d z$. (b) Integral of the transverse strain component over the spacer thickness, $\int\left(\partial u_{x} / \partial z\right) \cdot d z$. The profiles have been normalized to the maximum value.

equation, Eq. (2).

To investigate the effect of each SOC component individually, four modeled profiles have been plotted in Fig. 5. The model with the SOCs excluded (solid-red), the model with both SOCs (solid-black), the model with only the $p_{11}$ coefficient (dashed) and the model with only the $p_{12}$ coefficient. In the directivity equation the $p_{12}$ coefficient is multiplied by the integral of the normal strain over the thickness of the sensor. This is equivalent to taking the difference in the vertical displacement of the mirrors. Hence, after normalization to the normal-incidence response the dotted and solid red line overlap. The $p_{11}$ coefficient is multiplied by the integral of the transverse strain over the thickness of the sensor. As mentioned previously, the minima associated with the critical angle appears shifted. In Fig. 5, $p_{12}=0.3$ to exaggerate the effect. These results indicate strain-optic effect may not be significant for materials with large $p_{12}$ coefficients but will be most pronounced in materials with large $p_{11}$ coefficients.

\section{Discussion}

The results show that the inclusion of the SOCs in the modeled directivity do not make a significant change when compared with the measurements. This may not be true for all FP sensors. Firstly, the air-backed cover-slip sensor described in this study is planar. Other FP sensors are constructed on the end of optical fibers. In this case, a plane wave incident on 


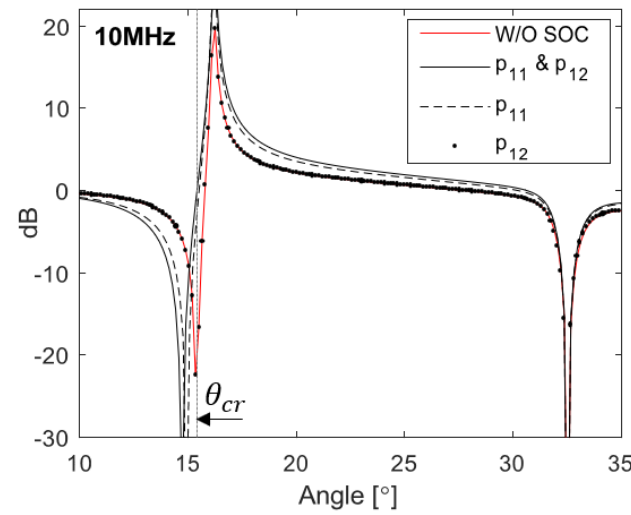

Fig. 5. Profiles at $10 \mathrm{MHz}$ when including and excluding different SOCs. The model with the SOCs excluded (solid-red), the model with both SOCs included (solid-black), the model with only the $p_{11}$ coefficient included (dashed) and the model with only the $p_{12}$ coefficient included.

such a sensor is diffracted around the tip which excites surface modes and tip resonances. The radial strains caused by these can have a significant strain-optic effect [10]. The geometry of the sensor in this study is simple, effectively consisting of one elastic layer, and the majority of features are associated with Lamb modes. Other FP sensors may be constructed from multiple elastic layers. The features in the directivity of these sensors will be different and include other types of guided modes. Additionally, the SOCs for each material may have to be considered in the transduction mechanism [3], [10]. The materials used for the FP sensors may have a significant impact on the strain-optic effect. Soft-polymer FP sensors commonly use Parylene C and PMMA as the substrate and spacer materials. The SOCs of PMMA can be seen in Table II. Note the $p_{11}=0.3$ and is larger than the equivalent SOC of glass. Values for Parylene C SOCs are not well known, however, if they are similar to PMMA the strainoptic effect would be more pronounced for soft-polymer planar sensors than glass sensors. Additionally, the acoustic and optical properties of thin films are not always well known and can often be optically or acoustically anisotropic. Acoustically anisotropic materials might increase the transverse strain and the effect of the $p_{11}$ coefficient. For example, a plane acoustic wave at normal incidence could cause a transverse motion. For optically anisotropic materials there are additional terms in the $\Delta n$ equation, for a laser beam parallel to $z$ and polarized in $x$

$$
\Delta n=-\frac{1}{2} n_{0}^{3}\left(p_{11} \frac{\partial u_{x}}{\partial x}+p_{12} \frac{\partial u_{z}}{\partial z}+p_{15} \frac{\partial u_{z}}{\partial x}\right) .
$$

The additional term introduces the shear-strain into the directivity equation $\partial u_{z} / \partial x$ and multiplies it by the $p_{15}$ SOC. Further study is needed to accurately determine the optical and acoustic properties of the materials used in other FP sensors, to have greater certainty into how significant the strain-optic effect is.

\section{CONCLUSiOnS}

A model of the directivity for a planar air-backed coverslip sensor has good agreement with measured data both with and without the inclusion of the SOCs. This suggests that the strain-optic effect is not significant for glass cover-slip sensors. However, this may not be true for all types of FP sensors. Understanding the underlying transduction mechanism of the FP sensor will help inform future sensor design.

\section{ACKNOWLEDGMENT}

We thank Paul Beard, Jamie Guggenheim, Elly Martin and Edward Zhang for their useful insights and discussion.

\section{REFERENCES}

[1] P. Beard and T. Mills, "Extrinsic optical-fiber ultrasound sensor using a thin polymer film as a low-finesse Fabry-Pérot interferometer," Appl. Optics, vol. 35, no. 4, pp. 663-675, 1996.

[2] P. Beard, F. Perennes, and T. Mills, "Transduction mechanisms of the Fabry-Pérot polymer film sensing concept for wideband ultrasound detection," IEEE Trans. Ultrason. Ferroelect. Freq. Contr., vol. 46, no. 6 , pp. 1575-1582, 1999.

[3] V. Wilkens, "Characterization of an optical multilayer hydrophone with constant frequency response in the range from 1 to $75 \mathrm{mhz}$," J. Acoust. Soc. Am., vol. 113, no. 3, pp. 1431-1438, 2003.

[4] B. Cox and P. Beard, "The frequency-dependent directivity of a planar Fabry-Pérot polymer film ultrasound sensor," IEEE Trans. Ultrason. Ferroelect. Freq. Contr., vol. 54, no. 2, 2007.

[5] E. Zhang, J. Laufer, and P. Beard, "Backward-mode multiwavelength photoacoustic scanner using a planar Fabry-Pérot polymer film ultrasound sensor for high-resolution three-dimensional imaging of biological tissues," Appl. Optics, vol. 47, no. 4, pp. 561-577, 2008.

[6] V. Wilkens and C. Koch, "Fiber-optic multilayer hydrophone for ultrasonic measurement," Ultrasonics, vol. 37, no. 1, pp. 45-49, 1999.

[7] V. Wilkens and C. Koch, "Amplitude and phase calibration of hydrophones up to $70 \mathrm{mhz}$ using broadband pulse excitation and an optical reference hydrophone," J. Acoust. Soc. Am., vol. 115, no. 6, pp. 28922903, 2004.

[8] K. Nakamura, Ultrasonic transducers: Materials and design for sensors, actuators and medical applications. Elsevier, 2012.

[9] D. Ramasawmy, E. Martin, J. Guggenheim, E. Zhang, P. Beard, B. Treeby, and B. Cox, "Analysis of the directivity of glass etalon Fabry-Pérot ultrasound sensors," IEEE Trans. Ultrason. Ferroelect. Freq. Contr., 2019.

[10] W. Weise, V. Wilkens, and C. Koch, "Frequency response of fiber-optic multilayer hydrophones: experimental investigation and finite element simulation," IEEE Trans. Ultrason. Ferroelect. Freq. Contr., vol. 49, no. 7, pp. 937-946, 2002.

[11] A. Ghatak and K. Thyagarajan, Optical Electronics. Cambridge University Press, 1989.

[12] J. Rose, Ultrasonic Waves in Solid Media, vol. 107. Cambridge University Press, 2004.

[13] M. Lowe, "Matrix techniques for modeling ultrasonic waves in multilayered media," IEEE Trans. Ultrason. Ferroelect. Freq. Contr., vol. 42, pp. 525-542, Jul 1995.

[14] J. Guggenheim, E. Zhang, and P. Beard, "A method for measuring the directional response of ultrasound receivers in the range $0.3-80$ mhz using a laser-generated ultrasound source," IEEE Trans. Ultrason. Ferroelect. Freq. Contr., vol. 64, no. 12, pp. 1857-1863, 2017.

[15] G. Hocker, "Fiber-optic sensing of pressure and temperature," Appl. optics, vol. 18, no. 9, pp. 1445-1448, 1979.

[16] N. Borrelli and R. Miller, "Determination of the individual strain-optic coefficients of glass by an ultrasonic technique," Appl. Optics, vol. 7, no. 5, pp. 745-750, 1968

[17] R. Waxler, D. Horowitz, and A. F.eldman, "Optical and physical parameters of plexiglas 55 and lexan," Appl. Optics, vol. 18, no. 1, pp. 101-104, 1979.

[18] M. Szczurowski, T. Martynkien, G. Statkiewicz-Barabach, L. Khan, D. Webb, C. Ye, J. Dulieu-Barton, and W. Urbanczyk, "Measurements of stress-optic coefficient and young's modulus in pmma fibers drawn under different conditions," in Photonic Crystal Fibers IV, vol. 7714, p. 77140G, International Society for Optics and Photonics, 2010. 